\title{
An integrated multidisciplinary model of COVID-19 recovery care
}

\author{
Helen O'Brien ${ }^{1}$ (D) - Michael J. Tracey ${ }^{2} \cdot$ Ciara Ottewill $^{1} \cdot$ Michael E. O'Brien ${ }^{1} \cdot$ Ross K. Morgan ${ }^{1} \cdot$ Richard W. Costello $^{1,3}$. \\ Cedric Gunaratnam ${ }^{1}$ - Daniel Ryan ${ }^{1}$ - Noel G. McElvaney ${ }^{1,3}$. Samuel J. McConkey ${ }^{2,4}$ - Cora McNally ${ }^{2}$. \\ Gerard F. Curley ${ }^{5} \cdot$ Siobhan MacHale $^{6,7} \cdot$ Diane Gillan $^{8} \cdot$ Niall Pender $^{8,9} \cdot$ Helen Barry $^{6,7} \cdot$ Eoghan de Barra ${ }^{2,4}$. \\ Fiona M. Kiernan ${ }^{5} \cdot$ Imran Sulaiman $^{1} \cdot$ Killian Hurley $^{1,3}$
}

Received: 14 July 2020 / Accepted: 25 August 2020 / Published online: 7 September 2020

(C) Royal Academy of Medicine in Ireland 2020

\begin{abstract}
Background In January 2020, the WHO declared the SARS-CoV-2 outbreak a public health emergency; by March 11, a pandemic was declared. To date in Ireland, over 3300 patients have been admitted to acute hospitals as a result of infection with COVID-19.

Aims This article aims to describe the establishment of a COVID Recovery Service, a multidisciplinary service for comprehensive follow-up of patients with a hospital diagnosis of COVID-19 pneumonia.

Methods A hybrid model of virtual and in-person clinics was established, supported by a multidisciplinary team consisting of respiratory, critical care, infectious diseases, psychiatry, and psychology services. This model identifies patients who need enhanced follow-up following COVID-19 pneumonia and aims to support patients with complications of COVID-19 and those who require integrated community care.

Results We describe a post-COVID-19 service structure together with detailed protocols for multidisciplinary follow-up. One hundred seventy-four patients were discharged from Beaumont Hospital after COVID-19 pneumonia. Sixty-seven percent were male with a median age (IQR) of 66.5 (51-97). Twenty-two percent were admitted to the ICU for mechanical ventilation, 11\% had non-invasive ventilation or high flow oxygen, and $67 \%$ did not have specialist respiratory support. Early data suggests that $48 \%$ of these patients will require medium to long-term specialist follow-up.

Conclusions We demonstrate the implementation of an integrated multidisciplinary approach to patients with COVID-19, identifying those with increased physical and mental healthcare needs. Our initial experience suggests that significant physical, psychological, and cognitive impairments may persist despite clinical resolution of the infection.
\end{abstract}

Keywords COVID-19 $\cdot$ Critical care $\cdot$ Multidisciplinary model $\cdot$ Psychiatry $\cdot$ Respiratory

Helen O'Brien

helenobrien@rcsi.ie

1 Department of Respiratory Medicine, Beaumont Hospital, Dublin, Ireland

2 Department of Infectious Diseases, Beaumont Hospital, Dublin, Ireland

3 Department of Medicine, Royal College of Surgeons in Ireland, Dublin, Ireland

4 Department of International Health and Tropical Medicine, Royal College of Surgeons in Ireland, Dublin, Ireland
5 Department of Anaesthesia and Critical Care, Beaumont Hospital, Dublin, Ireland

6 Department of Liaison Psychiatry, Beaumont Hospital, Dublin, Ireland

7 Department of Psychiatry, Royal College of Surgeons in Ireland, Dublin, Ireland

8 Department of Psychology, Beaumont Hospital, Dublin, Ireland

9 Department of Psychology, Royal College of Surgeons in Ireland, Dublin, Ireland 


\section{Introduction}

In December 2019, a cluster of cases of severe acute viral pneumonia were first described in Wuhan, China. In January 2020, the World Health Organization (WHO) declared SARSCoV-2 infection (COVID-19) a public health emergency. To date, there have been over 10 million cases confirmed worldwide [1]. In Ireland, more than 3300 confirmed cases have required hospital admission, with over 430 of these requiring intensive care unit (ICU) support [2].

While the long-term respiratory and functional limitations associated with COVID-19 pneumonia are yet to be fully understood, evidence from SARS-CoV-1 and the Middle East Respiratory Syndrome coronavirus (MERS-CoV) show a persistent physiological impairment among affected patients, with some developing permanent respiratory complications such as pulmonary fibrosis [3] [4].

It is known that those who survive critical illness not only have significant mortality and morbidity in the short to medium term but also experience significant psychological and physical dysfunction in the longer term. The physical and psychological difficulties experienced by critical care survivors result in frequent readmissions to hospital and subsequent healthcare resource utilization [5-7].

ICU admission is associated with particularly high rates of mental health complications as $33-75 \%$ of critical care survivors have clinically significant psychiatric or cognitive difficulties 1 -year post-ICU stay $[8,9]$. Rates are likely to be even higher for those who experienced COVID-19 due to the particular stressors associated with this condition $[10,11]$. This will result in a significant need for integrated psychosocial assessment and follow-up care for patients discharged from critical care settings.

The RCSI group COVID recovery service was established to provide an enhanced follow-up service to organize essential medical care and rehabilitation for those admitted with COVID pneumonia. The goal of the service is to deliver high quality care, reduce duplication of services, and reduce waiting times for patients after COVID-19. The surge of patient presentations also poses the challenge of a surge of patients for follow-up; thus a novel approach was required.

The described service is a multidisciplinary assessment and treatment system which includes a virtual phone-based assessment of patients admitted to Beaumont Hospital with COVID pneumonia. The team is made up of respiratory, intensive care medicine, infectious diseases, and psychiatry and psychology services to deliver appropriate and effective treatment strategies for patients after admission for COVID-19 pneumonia.

During the initial pandemic surge, approximately 400 patients were required acute medical admission to Beaumont Hospital with COVID-19 infection and 220 for the management of severe (ward based) and critical illness (ICU) related to respiratory failure. It is anticipated that approximately half of these patients will need medium to long-term follow up in a COVID survivorship clinic. It is envisioned that a large proportion of patients will be discharged to the community for integrated care with their GP and community mental health services, or followed longitudinally by specialist services such as respiratory medicine.

\section{Aims of service}

The overall aim of the service is to create a safe, effective, and multidisciplinary approach for patient follow-up after a diagnosis of COVID-19 pneumonia. The service plan was based on best international practice and in accordance with the British Thoracic Society guidelines [12] on post-COVID-19 patient care. The specific goals of the service are to optimize the usage of outpatient services by utilizing virtual assessment for the majority of patients, to reduce the use of the emergency department for assessment or admission, and to minimize the impact on overstretched outpatient departments. The multidisciplinary and hybrid of virtual and in-person approach described here can also be used as an exemplar for integrated chronic disease management and mental health service delivery in any healthcare setting during and after the COVID-19 pandemic.

\section{Service description and overview}

One hundred seventy-four patients were discharged from Beaumont Hospital between March 15 and June 30, 2020, after treatment for COVID-19 pneumonia. Sixty-seven percent were male with a median age (IQR) of 66.5 (51-97). Twenty-two percent were admitted to the ICU for mechanical ventilation, $11 \%$ had non-invasive ventilation or high flow oxygen on a specialist respiratory ward, and the remaining $67 \%$ had their care on a medical ward without the need for specialist respiratory support.

The COVID recovery outpatient service consists of an enhanced and virtual pathway, supported by a weekly multidisciplinary meeting involving all supporting specialties (Fig. 1). Enrolment into the service is based on a diagnosis of pneumonia on the admission chest X-ray, with a positive PCR test or a clinical diagnosis of COVID-19. All patients have a follow-up chest X-ray, blood testing, and complete a standardized phone-based assessment of symptoms, mental health status, and quality of life. Subsequently, their cases are discussed at the COVID recovery multidisciplinary meeting (MDM). All patients who were admitted to the ICU, or who required noninvasive ventilation at ward level, have enhanced follow-up at an in-person clinic. Patients who were admitted to the ward, but did not require ICU admission or non-invasive ventilation, have a virtual follow-up and are discharged to community 


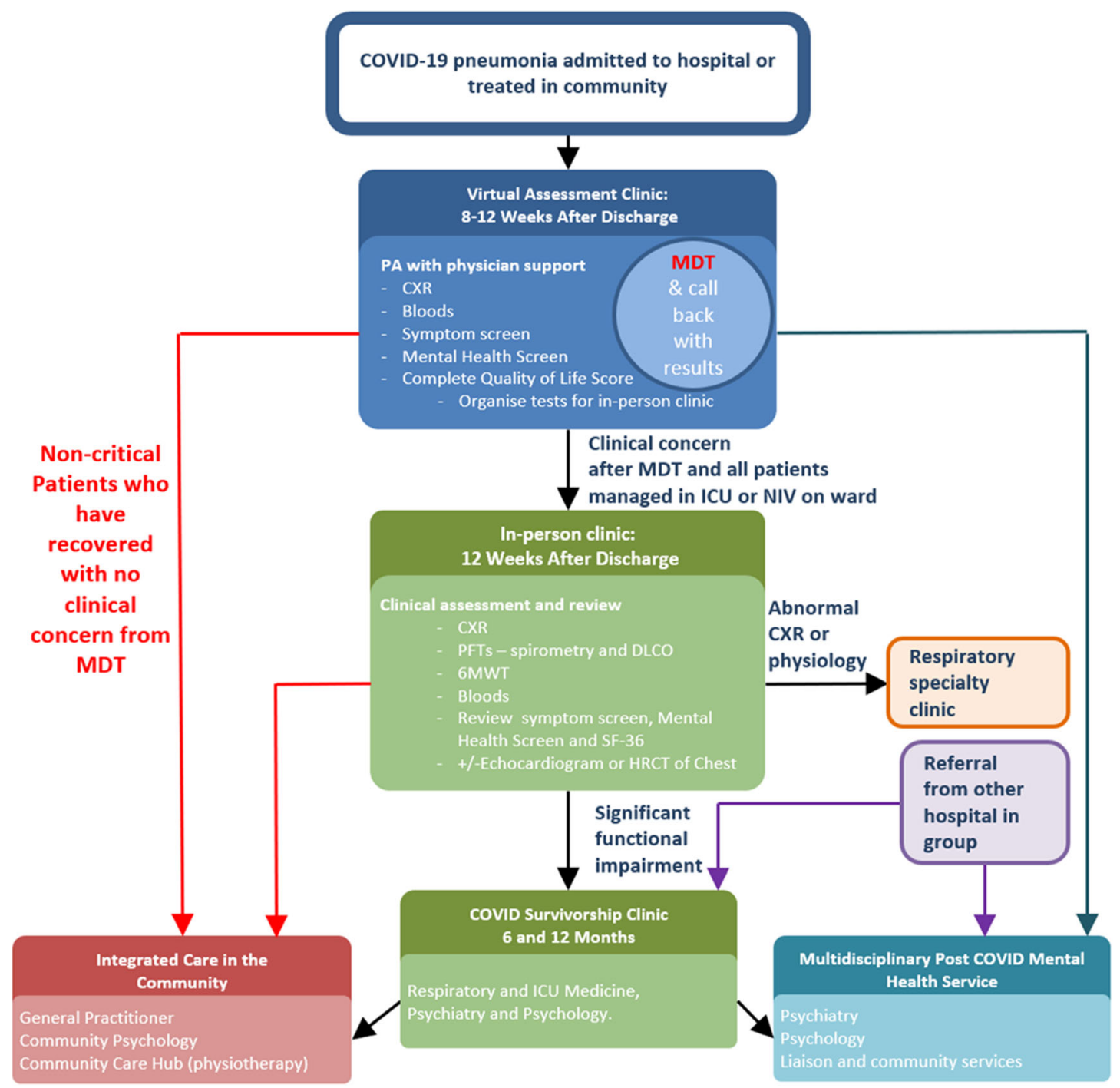

Intensive Care Unit (ICU); High Dependency Unit (HDU); Chest x-ray (CXR); multidisciplinary team (MDT); pulmonary function testing (PFT); 6-minute walk test (6MWT); short from health assessment (SF-36); high resolution computer tomography (CT).

Fig. 1 COVID recovery clinic clinical pathway. Patients that are hospitalized with COVID-19 pneumonia are followed up in an integrated multidisciplinary service. All patients receive a standardized assessment with a symptom and mental health screens and SF-36 quality of life

services unless a significant residual impairment is identified. This is then discussed at the COVID recovery MDM. This multidisciplinary approach encourages appropriate discharge to integrated community care with referral to relevant community services. Patients with greater medical, rehabilitation, and psychiatric and psychological needs will have an inperson assessment and may need a follow-up in a specialized survivorship clinic for 12 months.

\section{Virtual multidisciplinary assessment}

British Thoracic Society guidelines [12] recommend that all patients who were admitted to the hospital with COVID-19 pneumonia receive clinical follow-up regardless of severity. questionnaire. They also have a chest X-ray and blood tests. All patient cases are discussed at the COVID recovery multidisciplinary meeting, and a personalized plan is made

The RCSI COVID recovery service will carry out an initial assessment of all patients by phone at $8-12$ weeks postdischarge from the hospital or ICU. This virtual follow-up will ascertain their level of residual symptoms after COVID-19, functional capacity, quality of life scores, and the presence of mental health difficulties using a standardized questionnaire. The 36-Item Short Form Health Survey (SF-36) is a standardized evaluation of a patient's physical and mental health status and has previously been utilized as a tool to quantify disability for survivors of acute respiratory distress syndrome (ARDS) [13, 14].

This virtual clinic is run by a physician associate (PA) with oversight and governance from respiratory and infectious diseases, psychiatry, and ICU consultants. After initial basic 
investigations, including chest X-ray and blood tests (full blood count, renal, liver and bone biochemistry, BNP, D-dimer, and serum sample for SARS-CoV-2 antibody), each case is discussed at the COVID recovery MDM. A consensus is reached by the MDM to triage the patient to in-person or virtual follow-up and also if mental health follow-up is required. If no further specialist follow up is necessary, patients will be discharged to their GP with community follow-up as required.

Thus far, 50 patients have been reviewed at the MDM, 26/ 50 have been discharged to their GP, while 24/50 will require follow-up in the in-person COVID recovery clinic and with other specialty clinics.

\section{COVID recovery in-person clinic}

Patients identified as having persistent symptoms or radiographic changes at their initial virtual clinic review are offered an in-person clinic follow-up. Patients who were critically ill due to COVID-19, i.e., requiring ICU or noninvasive ventilation, are automatically offered an in-person follow-up at 12 weeks. This cohort of patients then receives an enhanced schedule of investigations including pulmonary function testing and 6-min walk testing, and some may require a CT thorax or an echocardiogram depending on clinical indications. All of these investigations will be reviewed at the COVID recovery MDM prior to being reviewed in the in-person clinic, where a personalized management plan is created for each patient. Patients with severe functional impairments may be referred onward to the COVID survivorship clinic or to a specialist clinic as deemed necessary. The COVID recovery clinic will also receive referrals from other RCSI group hospitals for discussion at the MDM.

\section{COVID survivorship clinic}

Established guidelines recommend that all critical care survivors should be reviewed 2-3 months after discharge, with the majority requiring a follow-up to at least 1 year $[15,16]$. The best practice post-ICU recommendations include a follow-up for all patients with an ICU stay of $>72 \mathrm{~h}$, selected patients with an ICU stay of $>48 \mathrm{~h}$, and any ICU patient with risk factors for psychological dysfunction [17]. Many COVID-19 survivors fulfill these criteria and are an at-risk group that requires follow-up.

Although it is difficult to quantify improvement in outcomes, qualitative studies have shown improvement for patients, and subjective outcomes studies have demonstrated improvements in SF36 scores, a surrogate for quality of life [18].
Fig. 2 Post-COVID mental health service screening questionnaire. Initial telephone triage tool to help identify patients at risk for mental health issues or who are currently experiencing mental health or cognitive symptoms. This system employs a traffic light system to help stratify patients and gains consent from the patient to be enrolled to a further in-depth cognitive and mental health assessment

\section{COVID recovery mental health service}

The COVID recovery service implements a brief telephone triage (Figs. 2 and 3), which includes both mental health and cognitive symptoms. Following the initial screening, those deemed in need of further mental health assessment and intervention are followed-up with stage 2 screening, which comprises of a telephone call and postal questionnaires from the COVID mental health service (COVPSYCH) team. The telephone call will include a brief objective and subjective screen of cognition. If the stage 2 screen is positive, a clinic or virtual appointment will be arranged with the COVPSYCH team within 4 weeks, whereby a full clinical assessment will be carried out, including cognitive testing where appropriate. If required, pharmacological and therapeutic intervention is instigated on a case-by-case basis.

\section{Discussion}

As a novel condition, the COVID-19 pandemic has presented clinicians with a unique challenge in regard to the appropriate follow-up of affected patients. Among the anticipated complications of COVID-19 infection include long-term respiratory impairment and post-ICU complications such as neuromuscular dysfunction, psychological dysfunction, and cognitive impairment. This has created the necessity for an integrated and comprehensive follow-up service, encompassing all members of the multidisciplinary model.

While the respiratory sequelae of COVID-19 are yet to be fully described, it is anticipated that patients will experience some degree of persistent respiratory symptoms or disease, reflecting the pattern of disease progression seen in the 2002-2004 SARS outbreak [19]. Prospective studies following healthcare workers with SARS-1 showed residual physiological impairment up to 2 years after infection [4]. This deficit was mainly demonstrated in the form of reduced gas transfer, with DLCO less than $80 \%$ of the predicted value in over $50 \%$ of patients studied.

There is emerging evidence that suggests that pulmonary thromboembolism is likely an under-reported morbidity associated with COVID-19 that carries a potentially significant burden of long-term complications including pulmonary hypertension [20]. In addition, previous studies have shown that acute lung injury is associated with pulmonary fibrosis on CT imaging and correlates with restrictive physiology and poorer 


\section{Post COVID Mental Health Service Screening Questionnaire}

MRN:

Date:

Referrer:

\section{Preferred contact number:}

Explicit consent to further screening obtained?

Stage 1: Initial Telephone Triage:

"I'm now going to ask you a couple of questions in relation to your mental health and psychological well-being"

A) Traffic Light Screen:

Q1. Do you have a post or present history of mental illness? $(\mathrm{Y} / \mathrm{N})$

Q2. Have you previously required inpatient care for your mental health ? $(\mathrm{Y} / \mathrm{N})$

Q3. Are you on medication for your mental health? $(\mathrm{Y} / \mathrm{N})$

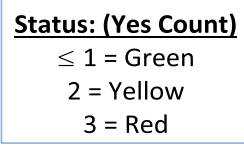

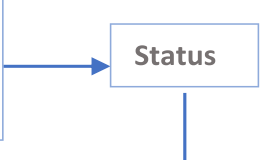

B) Depression Screen: "In relation to your mood..."

Q.4 During the last month, have you often been bothered by feeling down, depressed or hopeless? (YES/NO)

Q. 5. During the last month, have you often been bothered by little interest of pleasure in doing things? (YES/NO)

C) Anxiety Screen: "Over the last 2 weeks, how often have you

been bothered by the following problems?"

Q.6. Feeling nervous, anxious, or on edge $(0-3 ; 0=$ None $)$

Q.7. Not being able to stop or control worrying? (0-3)

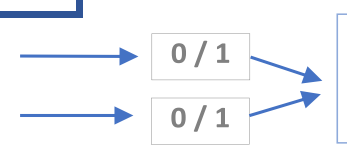

A cumulative total score $>\mathbf{3}$ for $\mathbf{Q} . \mathbf{6}$ \& Q.7 is the cut-off

D) Cognitive Screen: "During the past 2 weeks, how much (or how often) have you been bothered by:

Q. 8. Problems with memory (e.g., learning new information) or with location e.g., finding your way home)?"

\begin{tabular}{|l|l|l|l|l|}
\hline None & Slight & Mild & Moderate & Severe \\
\hline Not at all & Rare & Several says & $\begin{array}{l}\text { More than half } \\
\text { the days }\end{array}$ & $\begin{array}{l}\text { Nearly } \\
\text { everyday }\end{array}$ \\
\hline 0 & 1 & 2 & 3 & 4 \\
\hline
\end{tabular}

Q. 9 "Are you happy to be contacted by the COVID Mental Health Service in Beaumont Hospital to follow-up on these concerns with your emotions and/or memory or concentration problems?"

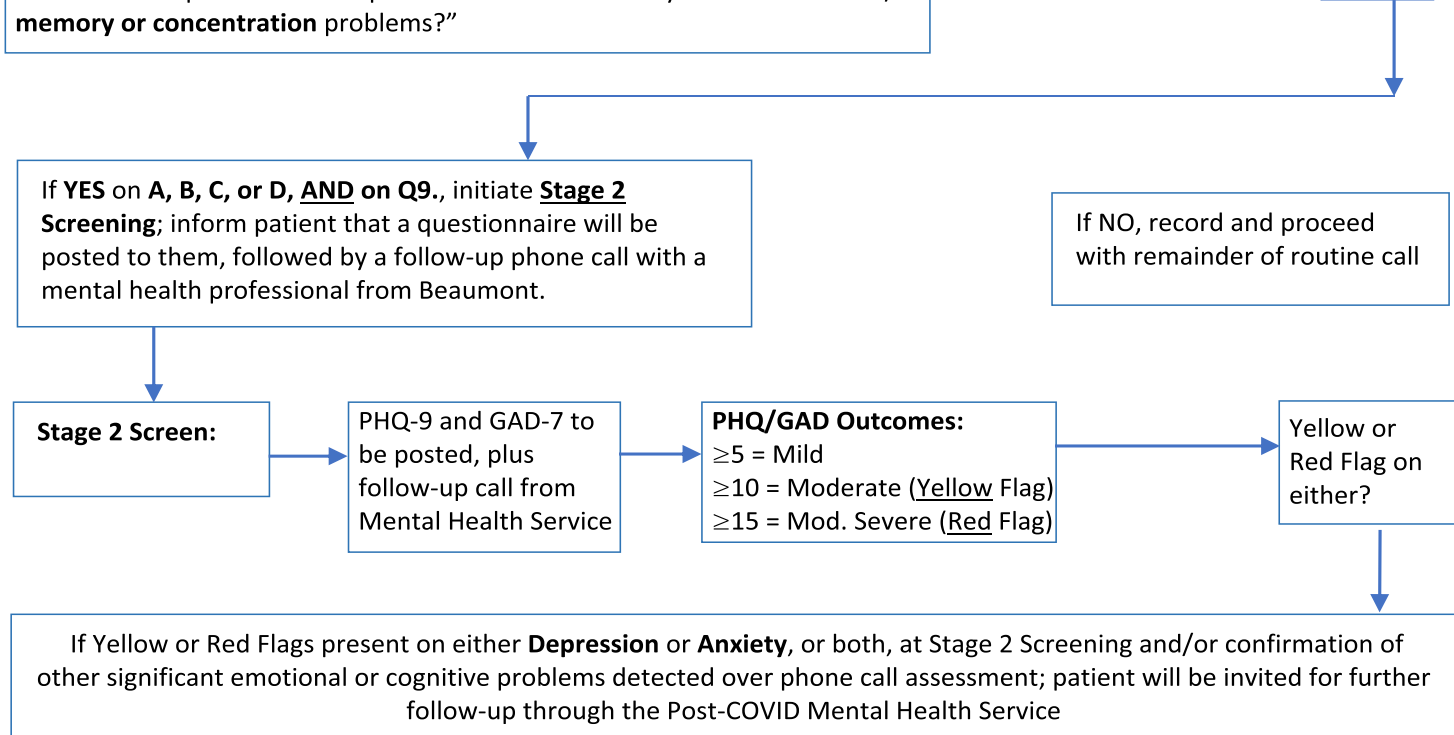


Pathway from Post COVID Clinic to Post COVID Mental Health Service

Stage 1: Telephone Triage

1. Previous mental health $h x$

2. Depression Screen

3. Anxiety Screen

4. Cognitive screen

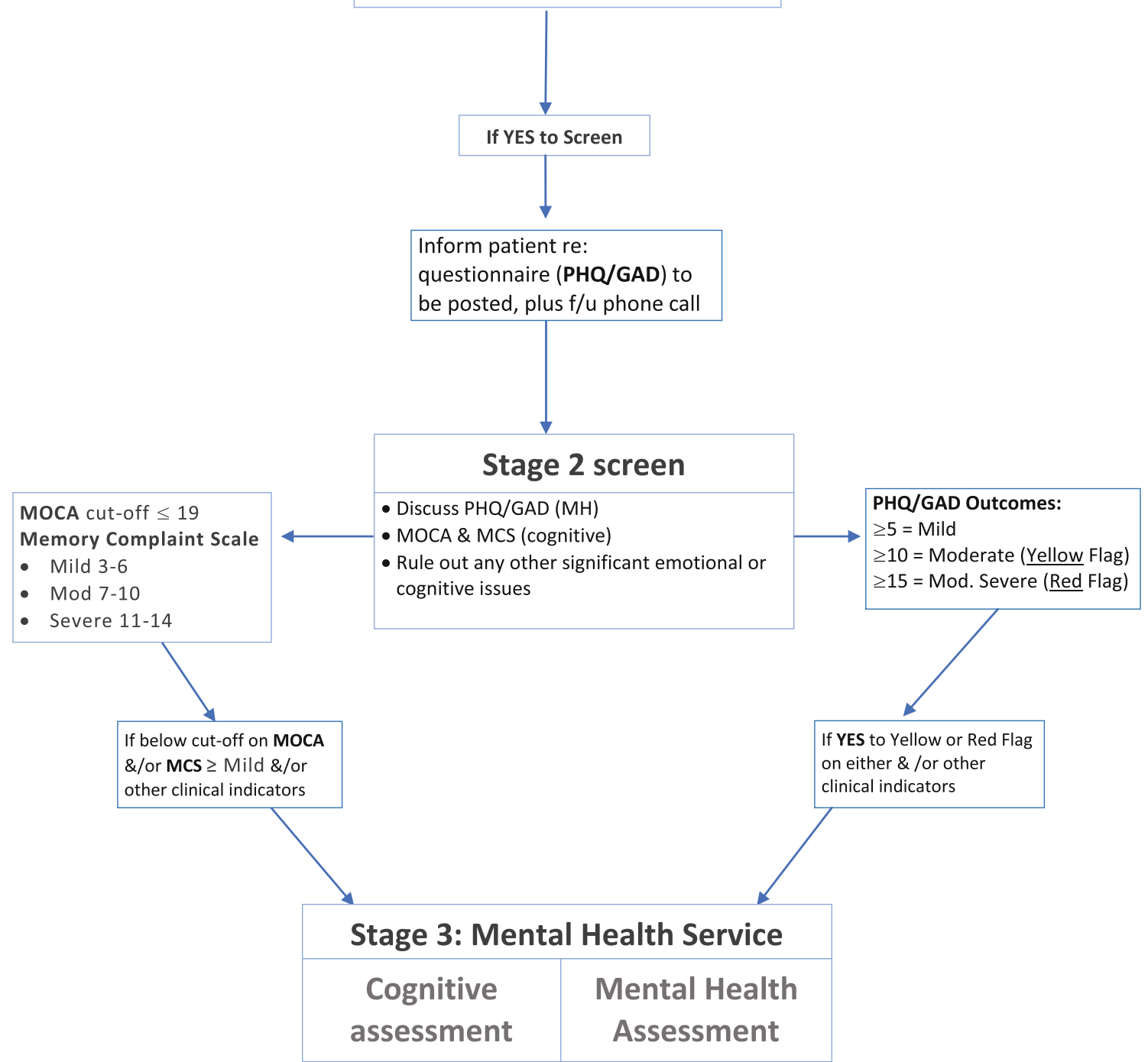

Fig. 3 Description of multidisciplinary model for mental health assessment. A staged mental health assessment consisting initially of a virtual triage assessment. Patients at risk for mental health or cognitive issues continue to stage 2 of the model where they have an in-depth assessment in person and proceed to a long-term follow-up as appropriate health-related quality of life [21]. These possible consequences of COVID-19 pneumonia highlight the unique challenge facing clinicians in the immediate aftermath of the pandemic surge due to restricted access to diagnostic imaging and pulmonary function testing due to infection control guidelines. This is an important factor to consider within the practicalities of any clinic structure and highlights the importance of the multidisciplinary model for decision-making purposes.

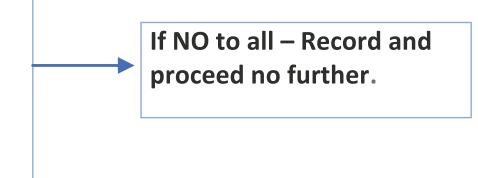


with longitudinal improvements in outcomes at 2.5-month intervals [22].

Mental health comorbidity in the acute hospital setting is associated with significant adverse consequences including increased health service and other costs, prolonged length of stay, earlier readmission rates, more frequent emergency department attendances, and poorer health outcomes [23]. COVID-19 is associated with a significant mental health burden in both the acute phase and the long term [24]. Anxiety, depression, insomnia, and post-traumatic stress disorder are common following severe coronavirus infections $[25,26]$. One-third of the first 153 COVID-19 cases reported in the UK were diagnosed with mental health problems including psychosis, cognitive decline, and affective disorder [24].

\section{Challenges of implementation}

Establishing a novel service of this nature presents its own challenges in the context of an already overstretched healthcare system. Resource management and the identification of additional staff, including a dedicated PA, have allowed for appropriate and timely follow-up and created a streamlined process for all patients. The coordination and cooperation of various departments including radiology, pulmonary physiology, phlebotomy, psychiatry, and psychology are integral to the success of instituting a multidisciplinary service such as this.

Some barriers we have encountered thus far are mainly focused on communication, particularly within the confines of a virtual clinic. We recognize that limitations exist in assessing patients over the phone, particularly in regard to language barriers with patients who do not speak English as their first language. While the SF-36 is an excellent tool to assess functional and cognitive limitations following an acute illness, patients may be reluctant to discuss sensitive mental health questions over the phone. As a result, we may underestimate the burden of physical and mental health issues in this cohort.

The practicalities of organizing an in-person clinic for any patient group during the rapidly evolving phases of a pandemic are challenging. Social distancing has reduced the volume of patients we can assess in one sitting and creates difficulties for patients navigating the hospital for their various investigations. Outsourcing of some initial investigations to satellite sites has helped to ensure that all relevant investigations are completed in accordance with our scheduling guidelines. However, we have already seen some non-attendances in our critical patient group as a result of mental and physical barriers post COVID-19.

\section{Conclusions}

In summary, we have demonstrated the design and implementation of an integrated multidisciplinary service for patients after admission to hospital with COVID-19 pneumonia. We have already shown that this service can assess patients using a hybrid of virtual and in-person clinics and assign appropriate follow-up for patients with complex physical and mental health needs. It is anticipated that this timely and comprehensive approach should lead to better outcomes for a novel disease with little precedent in the literature or clinical practice in Ireland or internationally. We envisage that our approach to COVID-19 recovery will improve the overall quality of patient care and strengthen patient acceptance for enhanced follow-up that may the subject of future longitudinal studies.

Acknowledgments We would first like to acknowledge the contribution of patients with COVID-19 who attended Beaumont Hospital and RCSI group hospitals initially and during follow-up clinics; without their generous contribution of time and feedback, we would not have been able to design and implement this novel service. We would also like to acknowledge the help and support of all of the COVID-19 pod team, medical, radiology, nursing and paramedical teams taking care of patients and acknowledge the essential role of hospital management and administrators in making this service possible. We thank Louise Clarke and her colleagues in the pulmonary function lab for their contribution.

Authors' contributions KH, IS, FK, EDB, HB, and NP conceived the clinic structure and protocols and contributed to writing the manuscript. $\mathrm{HOB}$ researched the literature and wrote the first draft of the manuscript. All authors critically revised and approved the final version of the manuscript.

\section{References}

1. WHO Coronavirus Disease (COVID-19) Dashboard [Internet]. [cited 2020 Jun 29]. Available from: https://covid19.who.int

2. Ireland's COVID19 Data Hub [Internet]. [cited 2020 Jun 29]. Available from: https://covid19ireland-geohive.hub.arcgis.com/

3. Hui D, Joynt G, Wong K, Gomersall C, Li T, Antonio G et al (2005) Impact of severe acute respiratory syndrome (SARS) on pulmonary function, functional capacity and quality of life in a cohort of survivors. Thorax. 60(5):401-409

4. Ngai JC, Ko FW, Ng SS, To K-W, Tong M, Hui DS (2010) The long-term impact of severe acute respiratory syndrome on pulmonary function, exercise capacity and health status. Respirol Carlton Vic 15(3):543-550

5. Prescott HC, Langa KM, Iwashyna TJ (2015) Readmission diagnoses after hospitalization for severe sepsis and other acute medical conditions. JAMA. 313(10):1055-1057

6. Unroe M, Kahn JM, Carson SS, Govert JA, Martinu T, Sathy SJ, Clay AS, Chia J, Gray A, Tulsky JA, Cox CE (2010) One-year trajectories of care and resource utilization for recipients of prolonged mechanical ventilation: a cohort study. Ann Intern Med 153(3):167-175

7. Ruhl AP, Huang M, Colantuoni E, Karmarkar T, Dinglas VD, Hopkins RO et al (2017) Healthcare utilization and costs in ARDS survivors: a 1-year longitudinal national US multicenter study. Intensive Care Med 43(7):980-991

8. Parker AM, Sricharoenchai T, Raparla S, Schneck KW, Bienvenu OJ, Needham DM (2015) Posttraumatic stress disorder in critical illness survivors: a metaanalysis. Crit Care Med 43(5):1121-1129

9. Rabiee A, Nikayin S, Hashem MD, Huang M, Dinglas VD, Bienvenu OJ, Turnbull AE, Needham DM (2016) Depressive 
symptoms after critical illness: a systematic review and meta-analysis. Crit Care Med 44(9):1744-1753

10. Rattray JE, Johnston M, Wildsmith JAW (2005) Predictors of emotional outcomes of intensive care. Anaesthesia. 60(11):1085-1092

11. Davydow DS, Desai SV, Needham DM, Bienvenu OJ (2008) Psychiatric morbidity in survivors of the acute respiratory distress syndrome: a systematic review. Psychosom Med 70(4):512-519

12. COVID-19: information for the respiratory community | British Thoracic Society | Better lung health for all [Internet]. [cited 2020 Jun 30]. Available from: https://www.brit-thoracic.org.uk/about-us/ covid-19-information-for-the-respiratory-community/

13. Herridge MS, Tansey CM, Matté A, Tomlinson G, Diaz-Granados N, Cooper A, Guest CB, Mazer CD, Mehta S, Stewart TE, Kudlow P, Cook D, Slutsky AS, Cheung AM, Canadian Critical Care Trials Group (2011) Functional disability 5 years after acute respiratory distress syndrome. N Engl J Med 364(14):1293-1304

14. Li TS, Gomersall CD, Joynt GM, Chan DPS, Leung P, Hui DSC (2006) Long-term outcome of acute respiratory distress syndrome caused by severe acute respiratory syndrome (SARS): an observational study. Crit Care Resusc J Australas Acad Crit Care Med 8(4): 302-308

15. Griffiths JA, Barber VS, Cuthbertson BH, Young JD (2006) A national survey of intensive care follow-up clinics. Anaesthesia. 61(10):950-955

16. Stevens RD, Hart N, Herridge MS (2014) Textbook of Post-ICU Medicine. The Legacy of Critical Care. Oxford University Press, $649 \mathrm{p}$

17. Van Der Schaaf M, Bakhshi-Raiez F, Van Der Steen M, Dongelmans DA, De Keizer NF (2015) Recommendations for intensive care follow-up clinics; report from a survey and conference of Dutch intensive cares. Minerva Anestesiol 81(2):135-144

18. Jones C, Skirrow P, Griffiths RD, Humphris GH, Ingleby S, Eddleston J, Waldmann C, Gager M (2003) Rehabilitation after critical illness: a randomized, controlled trial. Crit Care Med 31(10):2456-2461

19. Venkataraman T, Frieman MB (2017) The role of epidermal growth factor receptor (EGFR) signaling in SARS coronavirus-induced pulmonary fibrosis. Antivir Res 143:142-150

20. Wichmann D, Sperhake J-P, Lütgehetmann M, Steurer S, Edler C, Heinemann A, et al. Autopsy findings and venous thromboembolism in patients with COVID-19. Ann Intern Med 2020 6;

21. Burnham EL, Hyzy RC, Paine R, Coley C, Kelly AM, Quint LE et al (2013) Chest CT features are associated with poorer quality of life in acute lung injury survivors. Crit Care Med 41(2):445-456

22. Lasiter S, Oles SK, Mundell J, London S, Khan B (2016) Critical care follow-up clinics: a scoping review of interventions and outcomes. Clin Nurse Spec CNS 30(4):227-237

23. Unützer J, Schoenbaum M, Katon WJ, Fan M-Y, Pincus HA, Hogan D et al (2009) Healthcare costs associated with depression in medically ill fee-for-service medicare participants. J Am Geriatr Soc 57(3):506-510

24. Varatharaj A, Thomas N, Ellul M, Davies NW, Pollak T, Tenorio EL, et al. UK-wide surveillance of neurological and neuropsychiatric complications of COVID-19: the first 153 patients [Internet]. Rochester, NY: Social Science Research Network; 2020 May [cited 2020 Jun 29]. Report No.: ID 3601761. Available from: https:/ papers.ssrn.com/abstract=3601761

25. Kotfis K, Williams Roberson S, Wilson JE, Dabrowski W, Pun BT, Ely EW (2020) COVID-19: ICU delirium management during SARS-CoV-2 pandemic. Crit Care Lond Engl 24(1):176

26. Zambrelli E, Canevini M, Gambini O, D'Agostino A (2020) Delirium and sleep disturbances in COVID-19: a possible role for melatonin in hospitalized patients? Sleep Med 70:111

Publisher's note Springer Nature remains neutral with regard to jurisdictional claims in published maps and institutional affiliations. 